\title{
Explosive instability of geostrophic vortices. Part 1: baroclinic instability
}

\author{
Xavier Carton ${ }^{1,{ }^{*}}$, Glenn R. Flierl ${ }^{3}$, Xavier Perrot ${ }^{2}$, Thomas Meunier ${ }^{2}$ and Mikhail A. Sokolovskiy ${ }^{4}$
}

\author{
${ }^{1}$ LPO, UMR 6523, UEB/UBO, Brest, France \\ 2 LPO, UEB/UBO, Brest, France \\ 3 EAPS, MIT, Cambridge, MA, USA \\ ${ }^{4}$ Institute for Water Problems of the RAS, Moscow, Russia \\ *: Corresponding author : X. Carton, email address : xcarton@univ-brest.fr
}

\begin{abstract}
:
In a quasi-geostrophic model, we study the baroclinic instability of a two-layer vortex. The singular unstable modes for potential vorticity anomalies are compared with the classical normal modes. Shorttime singular modes are explosively unstable and, at short times, depend only on the baroclinic component of the flow. As time progresses, they evolve towards the normal modes and their sensitivity to flow parameters is explored. Asymptotic solutions are provided.
\end{abstract}

Keywords: Quasi-geostrophic equations - Inviscid flows - Vortex stability - Normal or singular modes Parametric resonance

PACS 92.05.Bc - 92.10.ak - 92.10.ei - 47.15 - 47.20.Cq 


\section{Introduction}

In stratified rotating turbulence and in planetary fluids, baroclinic processes have been recognized as essential in the generation and evolution of vortices. Indeed, such vortices can be generated by the instability of intense surface jets, or via mechanical or thermal forcing at the fluid surface. Once formed, these vortices most often reach a cyclogeostrophic balance in stratified, rotating fluids. Up to now, thestability of circular geostrophic vortices has been mostly studied with normal-mode perturbations ([1], [2], [3], [4], [5], [6], [7]). These studies have shown that the nonlinear evolution of such unstable vortices, perturbed with normal modes, can lead to more complex vortices (multipoles). Explosive growth of perturbations on atmospheric flows has often been observed. Such explosive growth is usually related to singular modes, which are the temporarily fastest growing perturbation of the linearized dynamical equations, ([8]). Singular modes are a linear combination of the normal modes when these latter are not orthogonal (i.e. when the linear operator is not self-adjoint). The present study considers the nature and properties of singular modes in the baroclinic instability of a circular vortex in a two-layer model, and relates them to normal modes. 


\section{Model equations, linear instability of the baroclinic vortex and normal modes}

The two-layer quasi-geostrophic equations describe the conservation of layerwise potential vorticity in the absence of forcing and of dissipation

$$
\frac{d q_{j}}{d t}=0, \quad \text { with } \quad \mathrm{q}_{\mathrm{j}}=\nabla^{2} \psi_{\mathrm{j}}+\mathrm{F}_{\mathrm{j}}\left(\psi_{\mathrm{k}}-\psi_{\mathrm{j}}\right)
$$

where $q_{j}$ is layerwise potential vorticity (the subscripts $j=1,2$ denote upper and lower layers respectively, and $k=3-j)), F_{j}$ are the layer coupling coefficients $\left(F_{j}=f_{0}^{2} / g^{\prime} H_{j}\right), H_{j}$ is layer thickness, and $H=H_{1}+H_{2}$. The internal deformation radius is $R_{d}=\sqrt{g^{\prime} H_{1} H_{2}} / f_{0} \sqrt{H}$ and $\gamma$ is its inverse.

In the two-layer quasi-geostrophic model, we study the instability of a circular baroclinic vortex, composed of two superimposed disks of constant potential vorticity $Q_{j}$ and of unit radius. This mean vortex is steady and has potential vorticity $\bar{Q}_{j}(r)$ and streamfunction $\bar{\psi}_{j}(r)$.

Hereafter, the perturbation is assumed to have a wave-like dependence in angle, but not always an exponential variation with time (i.e. the perturbation is not always a normal mode): its streamfunction is

$$
\psi_{j}^{\prime}(r, \theta, t)=A_{j}(t) \phi_{j}(r) \exp (i l \theta) .
$$

The perturbation is a displacement of the vortex boundary which becomes. This vortex boundary is then $r_{j}=1+\eta_{j}(\theta, t)$. The vorticity contour displacement is written

$$
\eta_{j}(\theta, t)=\eta_{j}^{0}(t) \exp (i l \theta) .
$$

The linear instability equations are obtained by applying three conditions : continuity of normal and tangential velocities at the boundary for the total flow, kinematic condition for the contour displacement (see again [1], [2], [3], [5]). For convenience, the linear equations are written in terms of barotropic and baroclinic flow components. These components are defined by $Q_{t}=h_{1} Q_{1}+h_{2} Q_{2}, Q_{c}=Q_{1}-Q_{2}$ with $h_{j}=H_{j} / H$. The mean flow has only tangential velocity given in barotropic and baroclinic components by $V_{t}(r)=Q_{t} r / 2, Q_{t} / 2 r$ (inside and outside the vortex) and $V_{c}(r)=Q_{c} I_{1}(\gamma r) K_{1}(\gamma), Q_{c} I_{1}(\gamma) K_{1}(\gamma r)$. We also write $\delta=H_{1} / H_{2}, \xi=(1-\delta) / \sqrt{\delta}$ and $V_{0}=V_{t}(1) / V_{c}(1)$. With these notations, the linear instability is described in vertical modes by the equations

$$
\begin{gathered}
\partial_{t} \eta_{t}^{0}=-i l\left[V_{t}(1)\left(1-\frac{1}{l}\right) \eta_{t}^{0}+V_{c}(1)\left(1-\frac{1}{2 l I_{1}(\gamma) K_{1}(\gamma)}\right) \eta_{c}^{0}\right] \\
\partial_{t} \eta_{c}^{0}=-i l\left[V_{c}(1)\left(1-\frac{I_{l}(\gamma) K_{l}(\gamma)}{I_{1}(\gamma) K_{1}(\gamma)}\right) \eta_{t}^{0}+V_{t}(1)\left(1-2 I_{l}(\gamma) K_{l}(\gamma)\right) \eta_{c}^{0}+\xi V_{c}(1)\left(1-\frac{I_{l}(\gamma) K_{l}(\gamma)}{I_{1}(\gamma) K_{1}(\gamma)}\right) \eta_{c}^{0}\right]
\end{gathered}
$$

This problem can be set in vector form as $\partial_{t} X=A X$ with $X\left(\eta_{t}^{0}, \eta_{c}^{0}\right)$ and

$A=-i l\left(\begin{array}{ll}a_{0} & b_{0} \\ c_{0} & d_{0}\end{array}\right)$

Normal modes are obtained by setting $\partial_{t} X=\sigma X$ where $\sigma$ contains the growth rate $s$ and the rotation rate $\omega$ of the perturbation $(\sigma=s-i l \omega)$. They are computed via

$$
\sigma_{ \pm}=\frac{-i l\left(a_{0}+d_{0}\right)}{2} \pm \frac{i l}{2} \sqrt{\Delta}
$$

with $\Delta=\left(a_{0}-d_{0}\right)^{2}+4 b_{0} c_{0}$. Instability occurs when $\Delta<0$.

These growth rates are plotted in the $\left(\gamma, V_{0}\right)$ plane in figure 1 (upper left panel) for $l=2$ and equal layer thicknesses $(\delta=1)$; one can clearly see that the largest growth rates are obtained for deformation radii smaller than the vortex radius $(\gamma>1)$ and for moderate barotropic potential vorticity of the mean state. Vertical symmetry of the physical configuration explains the invariance of results with respect to a sign reversal in $V_{0}$. For a shallower upper layer $\left(H_{1} / H_{2}=0.2\right.$, upper right panel of fig.1), this symmetry is broken and maximal growth rates are displaced towards negative components of barotropic potential vorticity of the mean state. Such vortices intensified at depths are not typical of the ocean. For very thin upper layers (not shown), the maximal growth rates are not substantially modified in amplitude, but they are shifted towards strongly negative $V_{0}$. Finally, for higher modes, maximal growth rates are similar in amplitude to those of $l=2$, but are found at larger deformation radii, as usual for baroclinic instability (see [3]). 

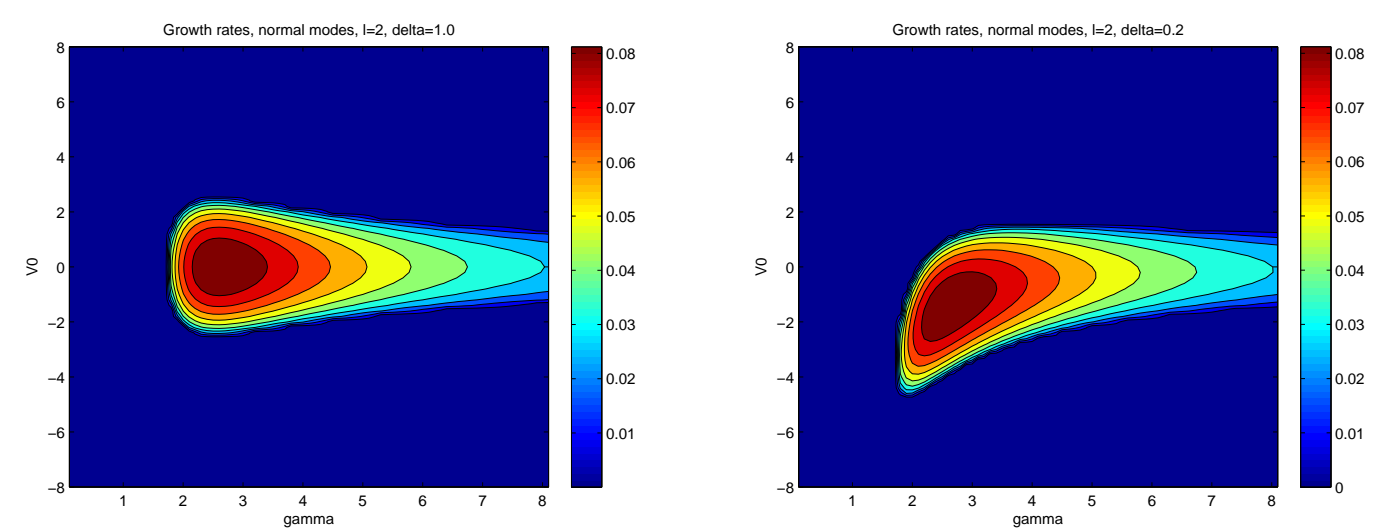

Fig. 1 Growth rates of normal modes in the $\left(\gamma, V_{0}\right)$ plane, for $l=2, \delta=1$ (left) and for $l=2, \delta=0.2$ (right)

\section{Singular modes definition and results and asymptotic formulations}

For the linear equation $\partial_{t} X=A X$ with $X\left(\eta_{t}^{0}, \eta_{c}^{0}\right)$, the solution will be

$$
X(t)=M(t) X(0), \quad M(t)=\exp (A t)
$$

if $A$ is stationary. $M$ is called the resolvent. Singular modes are defined as those providing the largest amplification rate at given time $t$; this rate is

$$
\lambda(t)=\frac{|X(t)|^{2}}{|X(0)|^{2}}=\frac{|M(t) X(0)|^{2}}{|X(0)|^{2}}
$$

Therefore, $\lambda(t)$ is the largest eigenvalue of $M^{*}(t) M(t)$ or $\exp \left(A^{*} t\right) \cdot \exp (A t)$. If $A$ is self-adjoint (Hermitian), then its eigenvectors (the normal modes) are orthogonal and identical to the singular modes, because then $M^{*} M=\exp (2 A t)$. Singular modes differ from normal modes when $A$ is not Hermitian. The anti-Hermitian part of the matrix is related to the baroclinic component of the mean state and to the different Green's functions for the Poisson and Helmholtz problems. Detailed calculation procedures for the singular modes and their associated eigenvectors are provided in [9] and [10]. These formulae have been adapted to the present problem.

For comparison with normal mode growth rates, we associate singular growth rates to the singular amplification rates via $\sigma_{s}(t)=\log (\lambda(t)) / 2 t$. Singular growth rates are plotted in figure 2 at different times. At short times, these rates do not depend on the barotropic component of the flow. Indeed, the onset of baroclinic instability depends on the sign reversal of the mean potential vorticity gradient (Charney-Stern criterion) and on the proper phase relation between layerwise perturbations. This involves only the baroclinic component of the flow. One can note also that singular growth rates are much larger then than those of normal modes (twice as large). This result can also be obtained via an asymptotic expansion of $M^{*} M$ at short times (as shown by [8] and by [9]):

$$
M^{*} M \sim I d+\left(A^{*}+A\right) t
$$

where $I d$ is the identity matrix. The amplification rates thus obtained are identical to those obtained by the complete computation of eigenvalues of $M^{*} M$ at short time (see again upper left panel of fig.2). This expansion also explains mathematically the independence of $\lambda$ on $V_{0}$ in this case: the extradiagonal terms involve only $V_{c}$ and not $V_{t}$. Now, as time goes, the diagonal terms of $M^{*} M$ will come into play and sensitivity to $V_{0}$ will appear. This is shown on figure 2, upper right and lower panels, for increasing time. A decrease in amplification rate accompanies this evolution. At long times, the singular growth rates become comparable to the normal mode growth rates. Indeed, as shown by [10], and calling $\eta_{+}\left(1, a_{+}\right)$and $\eta_{-}\left(1, a_{-}\right)$the eigenvectors associated to $\sigma_{+}$and to $\sigma_{-}$, the amplification rate at long times can be expressed as

$$
\lambda(t)=\frac{\left(1+\left|a_{+}\right|^{2}\right)\left(1+\left|a_{-}\right|^{2}\right)}{\left|a_{+}-a_{-}\right|^{2}} \exp \left(2 \operatorname{Re}\left(\sigma_{+} t\right)\right)
$$



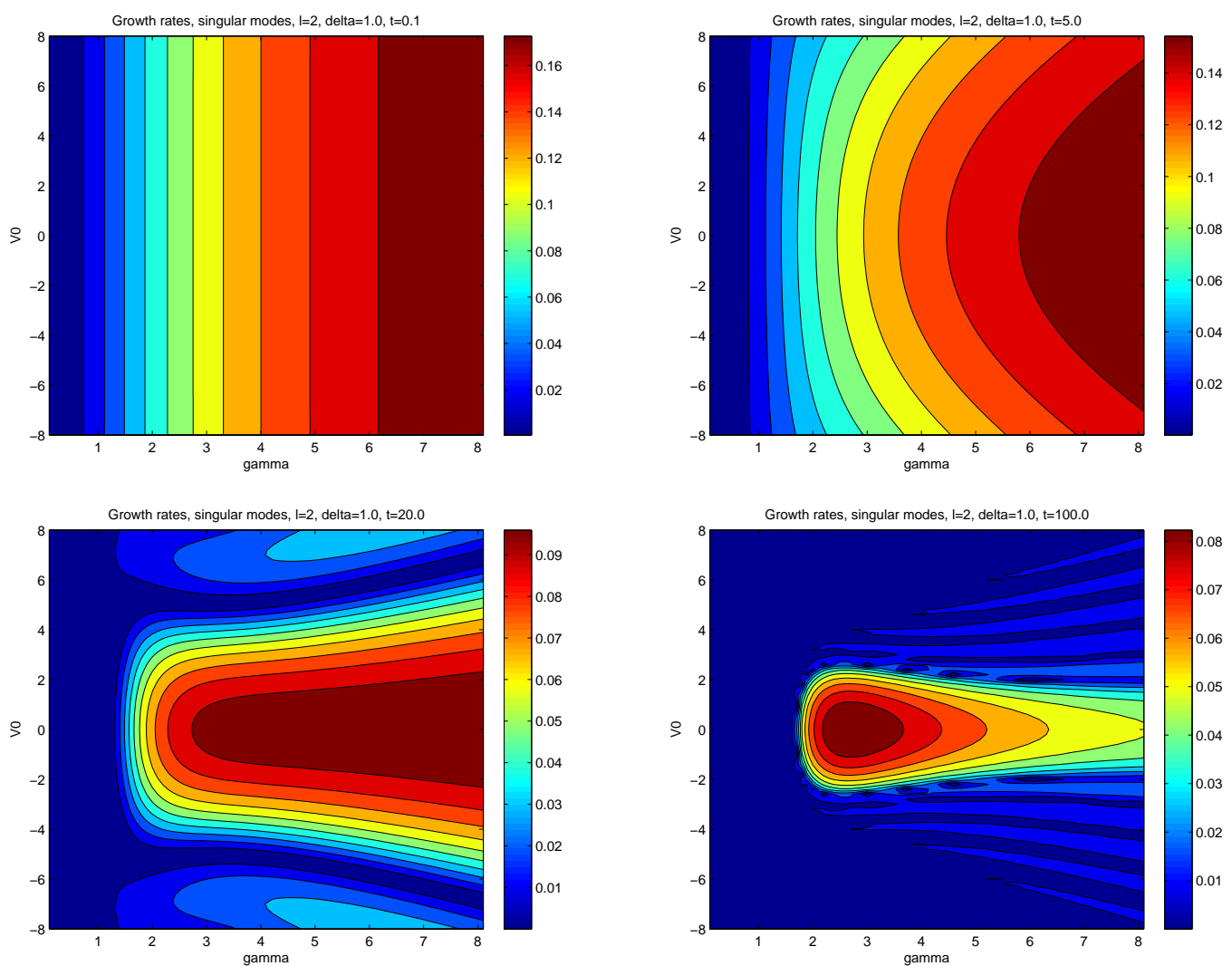

Fig. 2 Growth rates of singular modes at short time $(t=0.1$, upper left), and with longer time $(t=5,20,100$, upper right, lower left, lower right) in the $\left(\gamma, V_{0}\right)$ plane (case of $l=2$ and equal layer thicknesses).

and thus $\sigma_{s} \rightarrow \operatorname{Re}\left(\sigma_{+}\right)$(see again [9]). One can also note that the associated eigenvector is then the biorthogonal of $\eta_{+}$and is $\eta_{++}\left(a_{+},-1\right)$. Finally, the sensitivity of singular modes to physical parameters is explored. For higher modes than $l=2$, the time-evolution of singular modes is very similar to that shown on fig.2. For unequal layer thicknesses, the parity bias of growth rates with respect to $V_{0}$ grows with time as shown by fig.3, but the global evolution is similar to that of equal layer thicknesses.

\section{Conclusions}

In this paper, we have shown how a baroclinic vortex can be unstable both to normal and to singular modes in a two-layer quasi-geostrophic model. The sensitivity of baroclinic instability with normal modes to the barotropic component of the mean flow, to stratification and to the perturbation wavenumber have been explored. Singular modes can grow in regions of parameter space where normal modes are stable. Singular modes are due to the non-Hermiticity of the matrix associated with linear dynamics (or in other words to the absence of orthogonality of normal modes). Singular modes are much more unstable than normal modes, on short times. On the long run, their growth rates converge towards those of the normal modes.

\section{Acknowledgements}

$\mathrm{XC}$ and XP acknowledge fruitful discussions with Dr Bach Lien Hua during the course of this work.

\section{References}

1. Pedlosky, J.: The instability of continuous heton clouds. J. Atmos. Sci., 42, 1477-1486 (1985) 

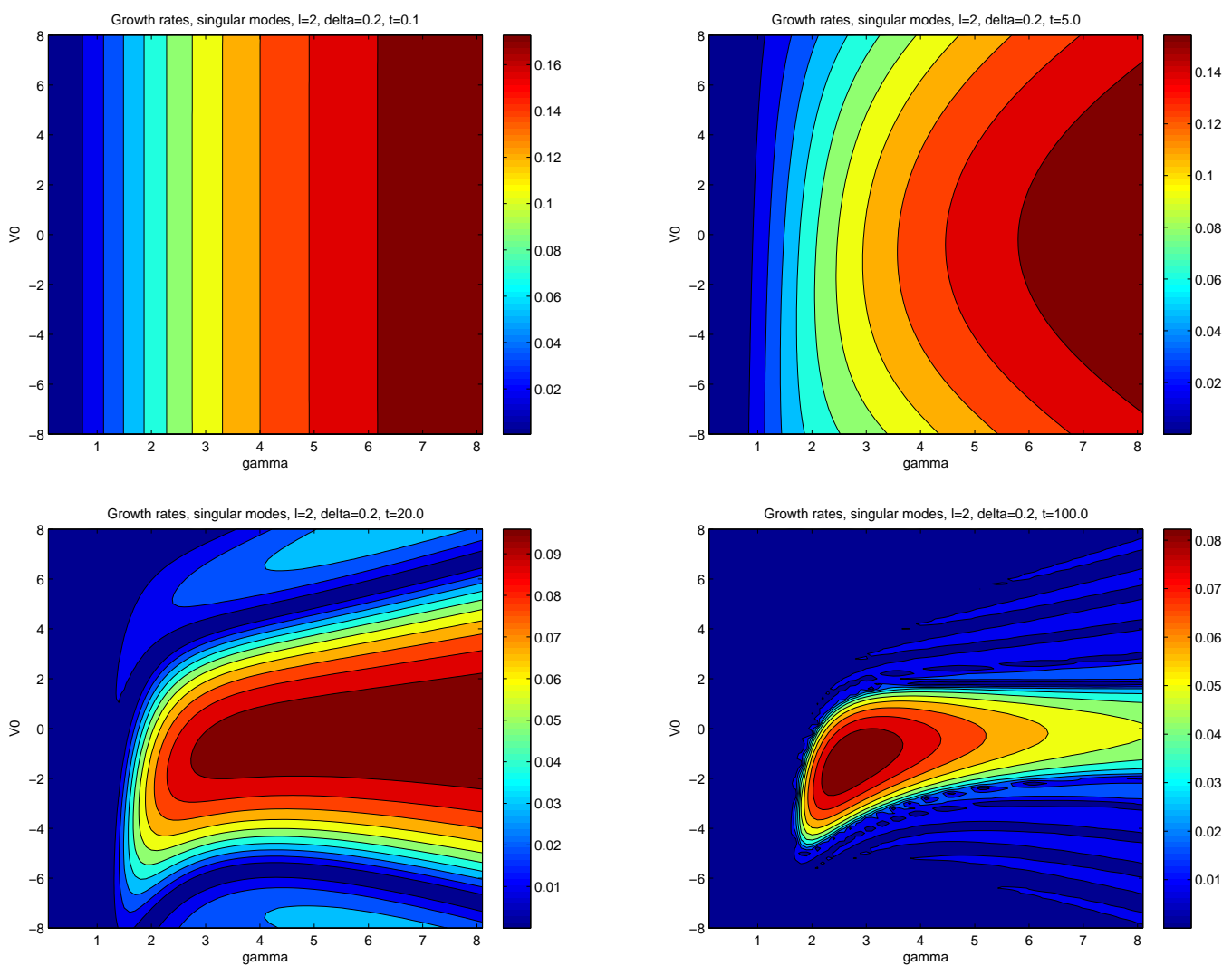

Fig. 3 Same as fig.2, for $\delta=0.2$

2. Kozlov, V.F., Makarov, V.G. and M.A. Sokolovskiy: A numerical model of baroclinic instability of axially symmetric vortices in a two-layer ocean. Izv. Atmos. Ocean. Phys., 22, 868-874 (1986)

3. Flierl, G.R.: On the instability of geostrophic vortices. J. Fluid Mech., 197, 349-388 (1988)

4. Helfrich, K.R. and U. Send: Finite-amplitude evolution of two-layer geostrophic vortices. J. Fluid Mech., 197, 331-348 (1988)

5. Sokolovskiy, M.A.: Numerical modelling on nonlinear instability of axially symmetric two-layered vortices. Izvestiya Atm. Ocean. Phys., 24, 735-743 (1988)

6. Carton, X.J. and J.C. Mc Williams: Barotropic and baroclinic instabilities of axisymmetric vortices in a QG model. In J.C.J. Nihoul and B.M. Jamart Eds., Mesoscale/Synoptic Coherent Structures in Geophysical Turbulence, Liège 1988 International Colloquium on Ocean Hydrodynamics, pp.225-244, Elsevier Oceanographic Series, 50 (1989)

7. Carton, X.J. and S.M. Corréard: Baroclinic tripolar vortices: formation and subsequent evolution. In J.N. Sorensen, E.J. Hopfinger and N. Aubry Eds, Simulation and identification of organized structures in flows, IUTAM/SIMFLOW 1997 Symposium in Lyngby, pp.181-190, Kluwer Acad. Publ. (1999)

8. Farrell, B.F. and P.J. Ioannou: Generalized stability theory. Part I: Autonomous Operators. J. Atmos. Sci., 53, 2025-2040 (1996a)

9. Riviere, G., Hua, B.L. and P. Klein: Influence of the beta-effect on non-modal baroclinic instability. Q. J. R. Meteorol. Soc., 127, 1375-1388 (2001)

10. Fischer, C.: Linear amplification and error growth in the $2 \mathrm{D}$ Eady problem with uniform potential vorticity. J. Atmos. Sci., 55, 3363-3380 (1998) 